\title{
Lax Pairs and First Integrals for Autonomous and Non-Autonomous Differential Equations Belonging to the Painlevé-Gambier List
}

\author{
P. Guha, S. Garai, A. G. Choudhury
}

Recently Sinelshchikov et al. [1] formulated a Lax representation for a family of nonautonomous second-order differential equations. In this paper we extend their result and obtain the Lax pair and the associated first integral of a non-autonomous version of the LevinsonSmith equation. In addition, we have obtained Lax pairs and first integrals for several equations of the Painlevé-Gambier list, namely, the autonomous equations numbered XII, XVII, XVIII, XIX, XXI, XXII, XXIII, XXIX, XXXII, XXXVII, XLI, XLIII, as well as the non-autonomous equations Nos. XV and XVI in Ince's book.

Keywords: Lax representation, Liénard type equations, Painlevé-Gambier equations, first integrals

Received June 21, 2020

Accepted September 17, 2020

Partha Guha

partha@bose.res.in

SN Bose National Centre for Basic Sciences

JD Block, Sector III, Salt Lake, Kolkata 700106, India

Department of Mathematics,

Khalifa University, Abu Dhabi, UAE

Sudip Garai

sudip.dhwu@gmail.com

A Ghose Choudhury

aghosechoudhury@gmail.com

Department of Physics, Diamond Harbour Women's University

D. H. Road, Sarisha 743368, West Bengal, India 


\section{Introduction}

A common feature of algebraically completely integrable (ACI) systems [2] is that they admit a one-parameter family of Lax representations. By an ACI system we mean that the system admits polynomial invariants, in sufficient number, and their (compact) invariant surfaces are real tori as given by the Arnold-Liouville theorem. In addition, there exists a transformation to the so-called action-angle variables, mapping the flow into a straight line motion on the torus $[2,3]$.

A Lax representation of a differential equation is a pair of matrices whose entries are meromorphic functions such that the differential equation may be obtained from the matrix equation

$$
\dot{L}(\lambda)=[L(\lambda), M(\lambda)] .
$$

Here $L(\lambda)$ and $M(\lambda)$ are finite Laurent series in the spectral parameter $\lambda$ whose coefficients are in $\mathfrak{g} \subset g l(n)$ and the over dot denotes the time derivative. The form of the Lax equation implies that, as time $t$ changes, the matrix $L(\lambda)$ remains in its coadjoint orbit:

$$
L(\lambda)(t)=(G(\lambda)(t)) L(\lambda)(0)(G(\lambda)(t))^{-1},
$$

so that the spectral curve is time-invariant.

In this paper we consider Lax pairs without the spectral parameter $\lambda$. This particular class of Lax pairs often appears in mechanics and finite-dimensional integrable systems. For instance, it may be verified that a suitable Lax pair for the equation of a linear harmonic oscillator is given by

$$
L=\left(\begin{array}{cc}
\dot{x} & x \omega \\
x \omega & -\dot{x}
\end{array}\right), \quad M=\left(\begin{array}{cc}
0 & \omega / 2 \\
-\omega / 2 & 0
\end{array}\right) .
$$

On the other hand, for the integrable case of the Hénon-Heiles equation Gravilov et al. [4] consider a Lax representation of the form

$$
L=\left(\begin{array}{cc}
F & U \\
W & -F
\end{array}\right), \quad M=\frac{1}{2 F}\left(\begin{array}{cc}
0 & \dot{U} \\
-\dot{W} & 0
\end{array}\right),
$$

where $U, F$, and $W$ are functions of $t$ with the property $U W+F^{2}=$ const.

The linearisation of the Lax flow can be described as follows. Consider an integrable system which linearises on a Jacobian variety $\operatorname{Jac}(\Gamma)$ of a hyperelliptic curve $\Gamma: \mu^{2}=f(\lambda)$, where $f(\lambda)$ is a polynomial with coefficients depending on the constants of motion. As first noted by Fairbanks, if we take $f(\lambda)=c$, where $c$ is a constant, then $U, F$, and $W$ are given by Jacobi polynomials on $\operatorname{Jac}(\Gamma)$.

Note that in [5] the form of the matrix $M$ is taken as

$$
\widetilde{M}=\frac{1}{U}\left(\begin{array}{cc}
0 & 0 \\
\dot{F} & -\dot{U}
\end{array}\right),
$$

which differs from that in [4] by a linear combination of $L$ and the identity matrix. Interestingly, the Lax representation for the Kovalevskaya top also follows from the construction of Fairbanks [5]. 
Recently Sinelshchikov et al. [1] have extended the Lax formalism to a family of nonautonomous second-order differential equations generalizing the Liénard equation [6]. This stands in contrast to the earlier works which dealt mostly with completely integrable systems. The work of Sinelshchikov et al. provides a connection between the existence of a quadratic first integral and a Lax representation for the dissipative differential equations studied by the authors. It also demonstrates that the notion of Lax integrability is not only valid for conservative Hamiltonian systems, but also for any system of differential equations.

The aim of this paper is to expand the scope of the Lax formulation method to deduce first integrals of autonomous and non-autonomous differential equations. In particular, we obtain the first integrals of several equations of the Painlevé-Gambier list, namely, equations Nos. XII, XV, XVI, XVII, XVIII, XIX, XXI, XXII, XXIII, XXIX, XXXII, XXXVII, XLI and XLIII [7]. All these first integrals are quadratic in nature unlike our previous results [8] where we obtained first integrals for a number of equations of the Painlevé-Gambier list using the generalised Sundman transformation method, none of which were quadratic.

\section{Lax Formulation for equations of Liénard-type}

Given a second-order non-linear ordinary differential equation of non-autonomous type, it is at times possible to obtain a Lax pair for the system. Recently Kudryashov and Sinelshchikov [6] have demonstrated a procedure for constructing a Lax pair for the non-autonomous Liénard equation

$$
\ddot{x}+f(x, t) \dot{x}+g(x, t)=0,
$$

which is briefly outlined below. By a Lax pair we mean the following system of linear equations:

$$
L \psi=\lambda \psi, \quad \psi_{t}+M \psi=0,
$$

whose consistency requires that the matrix operators $L$ and $M$ satisfy the following equation:

$$
L_{t}=[L, M] .
$$

It is also straightforward to show that, if a Lax pair exists, then one can obtain an infinite set of conserved quantities which are given by

$$
I_{k}=\frac{\operatorname{tr}\left(L^{k}\right)}{k !}
$$

For the Liénard equation the forms of the Lax matrices are taken as

$$
L=\left(\begin{array}{cc}
\dot{x}+F & U \\
U & -(\dot{x}+F)
\end{array}\right), \quad M=\left(\begin{array}{cc}
A & B \\
C & D
\end{array}\right) .
$$

Here the functions $F, U$ and the elements of the matrix $M$ are all assumed to be functions of $x, t$ and are to be determined. Inserting the matrices $L$ and $M$ in (2.3) and using the equation of motion (2.1), we find, on equating the powers of $\dot{x}$, that the entries of the matrices $L$ and $M$ satisfy the following set of equations:

$$
B=-C=\frac{1}{2} U_{x}, \quad U_{t}=2 B F, \quad F_{x}=f, \quad F_{t}-g=U(C-B) .
$$


Furthermore, it will be observed that one may choose without loss of generality $A=D=0$, whence the explicit form of $F$ is given by

$$
F(x, t)=\int^{x} f(\xi, t) d \xi+\mu(t)
$$

where $\mu$ is an arbitrary constant of integration. In order to mimic the standard autonomous form of the Liénard equation, we may assume $\mu=0$ and $f(x, t)=f(x)$, i.e., independent of $t$ so that $F(x)=\int^{x} f(\xi) d \xi$. Moreover, it will be observed that $U$ satisfies the linear PDE

$$
U_{t}-F(x) U_{x}=0
$$

which has the solution

$$
U(x, t)=\kappa\left(t+\int^{x} \frac{d \eta}{F(\eta)}\right),
$$

where $\kappa$ is an arbitrary function. As $F_{t}=0$, the functional form of $\kappa$ must be such as to satisfy the condition $g(x, t)=2 B U=U_{x} U$, which clearly demonstrates the non-autonomous character of the equation, for one cannot take the function $g$ to be time-independent. Consequently, given an equation of Liénard type, the functional form of $U$, i.e., the form of the function $\kappa$, is to be determined from the condition $U^{2}(x, t)=2 \int g(x, t) d x+\nu(t)$.

We now consider a modification of the $L$ part of the Lax pair in (2.5) to allow for the description of non-autonomous equations of Levinson-Smith type.

\subsection{Lax formulation of the non-autonomous Levinson-Smith equation}

A non-autonomous equation of Levinson-Smith type is characterised by a damping term (similar to the Liénard equation) along with a term quadratic in the velocity, and is given by

$$
\ddot{x}+f_{2}(x, t) \dot{x}^{2}+f_{1}(x, t) \dot{x}+g(x, t)=0 .
$$

In view of the foregoing results, we consider the following modification of the Lax pair:

$$
L=\left(\begin{array}{cc}
m(x) \dot{x}+F(x, t) & U(x, t) \\
U(x, t) & -(m(x) \dot{x}+F(x, t))
\end{array}\right), \quad M=\left(\begin{array}{cc}
0 & B \\
C & 0
\end{array}\right) .
$$

Here we have assumed $A=D=0$ in the light of our previous experience. Then from the Lax equation, $L_{t}=[L, M]$, it follows that

$$
\begin{gathered}
\frac{d}{d t}(m(x) \dot{x})+F_{x} \dot{x}+F_{t}=U(C-B), \\
U_{x}=2 B m(x), \quad \text { so that } \quad B=\frac{U_{x}}{2 m(x)}, \\
U_{t}=2 B F(x, t)=\frac{U_{x}}{m(x)} F(x, t), \\
C=-\frac{U_{x}}{2 m(x)} .
\end{gathered}
$$


Substituting (2.9) into the the first equation of the above set we have upon equating coefficients of the different powers of $\dot{x}$ the following equations:

$$
\begin{gathered}
m^{\prime}(x)=m(x) f_{2}(x, t) \quad \Rightarrow \quad m(x)=m_{0}(t) e^{\int^{x} f_{2}(\xi, t) d \xi}, \\
F_{x}=m(x) f_{1}(x, t), \Rightarrow F(x, t)=\int m(\xi) f_{1}(\xi, t) d \xi+\mu(t), \\
F_{t}-m(x) g(x, t)=-2 B(x, t) U(x, t)=-\frac{1}{m(x)} U U_{x}
\end{gathered}
$$

As before, let us suppose $F_{t}=0$, which implies that $U U_{x}=m^{2}(x) g(x, t)$, where we have used (2.12). On the other hand, from Eq. (2.12) we find

$$
U_{t}-\frac{F(x)}{m(x)} U_{x}=0
$$

which may be solved by the Lagrange method to yield

$$
U(x, t)=\kappa\left(t+\int^{x} \frac{m(\eta) d \eta}{F(\eta)}\right)
$$

The elements $B$ and $C$ of the matrix $M$ can then be obtained from the second and the fourth equations of the previous set and are given by

$$
B(x, t)=\frac{1}{2 F(x, t)} \frac{\partial \kappa}{\partial \eta}=-C(x, t)
$$

Also, both $f_{1}$ and $f_{2}$ may be taken to be independent of $t$, in which case we have

$$
\begin{gathered}
F(x, t)=\int^{x} m(\xi) f_{1}(\xi) d \xi \\
m(x)=e^{\int f_{2}(\xi) d \xi}
\end{gathered}
$$

The only non-autonomous term of the equation is then $g(x, t)$, which is given by

$$
g(x, t)=\frac{1}{m^{2}(x)} \frac{\partial}{\partial x}\left(\frac{U^{2}}{2}\right)
$$

as $U(x, t)$ is explicitly time-dependent. We illustrate the above procedure with an example:

Example. Let us assume a power-law form for $m(x)=x^{\alpha}$, so that $f_{2}(x, t)=\alpha / x$ and consider the generic equation

$$
\ddot{x}+\frac{\alpha}{x} \dot{x}^{2}+f_{1}(x, t) \dot{x}+g(x, t)=0 .
$$

The Lax matrix then has the form

$$
L=\left(\begin{array}{cc}
x^{\alpha} \dot{x}+F(x) & U(x, t) \\
U(x, t) & -\left(x^{\alpha} \dot{x}+F(x)\right)
\end{array}\right), \quad M=\left(\begin{array}{cc}
0 & B \\
-B & 0
\end{array}\right),
$$


where we have assumed $F$ to be independent of $t$. With this form of the Lax pair we obtain from the Lax equation the following set:

$$
U_{x}=2 B x^{\alpha}, \quad U_{t}=2 B F(x),
$$

which implies

$$
U(x, t)=\tilde{f}\left(t+\int \frac{x^{\alpha}}{F(x)} d x\right),
$$

with $\tilde{f}$ being an arbitrary function. The other relevant equation is given by

$$
x^{\alpha}\left(\ddot{x}+\frac{\alpha}{x} \dot{x}^{2}\right)+F_{x} \dot{x}=-\frac{U_{x} U}{x^{\alpha}} .
$$

Inserting the given equation, we see that, in order to match the coefficient of $\dot{x}$, we require

$$
\frac{F_{x}}{x^{\alpha}}=f_{1}(x, t) .
$$

Clearly, as we have taken $F$ to be independent of $t$, we restrict ourselves to functions $f_{1}$ which are also $t$-independent. For explicitness let us now assume $f_{1}(x, t)=q x^{\beta}$ where $q$ and $\beta$ are constants. The result of such a choice of the function $f_{1}$ is that the functional form of $F(x)$ and the characteristic coordinate $\eta$ are now explicitly given by

$$
F(x)=\frac{q}{\alpha+\beta+1} x^{\alpha+\beta+1}, \quad \eta=t-\frac{\alpha+\beta+1}{q \beta x^{\beta}} .
$$

The remaining $\dot{x}$ independent terms yield

$$
\frac{\partial}{\partial \eta} \widetilde{f}^{2}(\eta)=\frac{2 q}{(\alpha+\beta+1)} x^{2 \alpha+\beta+1} g(x, t) .
$$

To simplify matters further, let us require that $2 \alpha+\beta+1=0$, which yields

$$
\widetilde{f}^{2}(\bar{\eta})=-\frac{2 q}{\alpha} \int g(\bar{\eta}) d \bar{\eta}, \quad \bar{\eta}=t-\frac{\alpha}{q(2 \alpha+1)} x^{2 \alpha+1},
$$

provided we can write $g(x, t)=g(\bar{\eta})$. It follows that the simple choice $g(y)=\gamma y$ gives the equations

$$
\begin{gathered}
U(x, t)=\sqrt{-\frac{\gamma q}{\alpha}}\left(t-\frac{\alpha x^{2 \alpha+1}}{q(2 \alpha+1)}\right), \\
F(x)=-\frac{q}{\alpha x^{\alpha}}, \quad B(x)=-\frac{\alpha}{2 q} \sqrt{-\frac{\gamma q}{\alpha}} x^{\alpha},
\end{gathered}
$$

which completely determine the Lax pair and yield the following equation:

$$
\ddot{x}+\frac{\alpha}{x} \dot{x}^{2}+\frac{q}{x^{2 \alpha+1}} \dot{x}+\gamma\left(t-\frac{\alpha}{q(2 \alpha+1)} x^{2 \alpha+1}\right)=0 .
$$

One must note that, if we apply a folding transformation, $x=Q^{\frac{1}{\alpha+1}}$, then $(2.20)$ is transformed to a non-autonomous Liénard type equation

$$
\ddot{Q}+q \dot{Q} Q^{-\frac{2 \alpha+1}{\alpha+1}}+\widetilde{\gamma}\left(Q^{\frac{\alpha}{\alpha+1}} t-\frac{\alpha}{q(2 \alpha+1)} Q^{\frac{3 \alpha+1}{\alpha+1}}\right)=0 .
$$


Proposition 2.1 The Lax pair and the associated quadratic first integral of Eq. (2.20) are given by

$$
\begin{aligned}
& L=\left(\begin{array}{cc}
\dot{x} x^{\alpha} & {\left[\frac{2 \gamma}{2 \alpha+1}\left\{t-\frac{\alpha}{2 q} x^{2 \alpha+1}\right\}\right]^{\frac{1}{2}} x^{\alpha+\frac{1}{2}}} \\
{\left[\frac{2 \gamma}{2 \alpha+1}\left\{t-\frac{\alpha}{2 q} x^{2 \alpha+1}\right\}\right]^{\frac{1}{2}} x^{\alpha+\frac{1}{2}}} & -\dot{x} x^{\alpha}
\end{array}\right), \\
& 0=\left(\begin{array}{cc}
0 & \sqrt{\frac{\gamma(2 \alpha+1)}{2}} \frac{\left(t-\frac{\alpha}{q} x^{2 \alpha+1}\right)}{\sqrt{t-\frac{\alpha}{2 q} x^{2 \alpha+1}} x^{\alpha-\frac{1}{2}}} \\
-\sqrt{\frac{\gamma(2 \alpha+1)}{2}} \frac{\left(t-\frac{\alpha}{q} x^{2 \alpha+1}\right)}{\sqrt{t-\frac{\alpha}{2 q} x^{2 \alpha+1}} x^{\alpha-\frac{1}{2}}} & 0
\end{array}\right) .
\end{aligned}
$$

Finally, it may be checked that a first integral for Eq. (2.20) is given by

$$
I_{2}(x, t)=\dot{x}^{2} x^{2 \alpha}+\frac{2 \gamma}{2 \alpha+1}\left(t-\frac{\alpha}{2 q} x^{2 \alpha+1}\right) x^{2 \alpha+1}
$$

and is explicitly time-dependent.

\section{Lax pair of equations from the Painlevé-Gambier list}

The list of 50 Painlevé-Gambier equations representing all second-order ODEs which do not have movable critical points provides a rich ground for applications of the procedure outlined in the previous sections. Leaving aside the six Painleve equations of the remaining 44, there are several equations which are non-autonomous. In the following subsection we deduce Lax pairs and first integrals for some of these non-autonomous equations.

\subsection{Non-autonomous Painlevé-Gambier equations}

In this segment we consider some of the non-autonomous equations belonging to the PainlevéGambier list as given in [7].

\section{Equation XV}

$$
\ddot{x}=\frac{1}{x} \dot{x}^{2}+\frac{1}{x} \dot{x}+r(t) x^{2}-x \frac{d}{d t}\left(\frac{\dot{r}}{r}\right) .
$$

This belongs to the Levinson-Smith category. We assume the same form of the Lax pair as given in (2.10) with $C=-B$. Then, upon using the Lax equation, we arrive at the following:

$$
m(x)=\frac{1}{x}, \quad F(x, t)=\frac{1}{x}+s(t),
$$

where $s(t)$ is to be determined along with the following equations for determining $U$ and $B$ :

$$
\begin{gathered}
F_{t}+r(t) x-\frac{d}{d t}\left(\frac{\dot{r}}{r}\right)=-2 U B, \\
U_{t}=2 F(x, t) B, \quad U_{x}=\frac{2 B}{x}, \quad \text { which implies } U_{t}-(1+x s(t)) U_{x}=0 .
\end{gathered}
$$

The characteristic coordinate is found to be

$$
\eta=x e^{\int s(t) d t}+\int e^{\int s(t) d t} d t
$$


so that $U=\mathcal{F}(\eta)$. The remaining equation may be expressed as a function of the characteristic coordinate provided we choose $s(t)=\dot{r} / r$. This leads to the following functional form of $U$ :

$$
U(x, t)=\sqrt{-2 \eta}, \quad B=-\frac{x r(t)}{2 \sqrt{-2 \eta}}, \quad \text { where } \quad \eta=x r(t)+\int r(t) d t .
$$

The Lax pair is therefore

$$
\begin{aligned}
L & =\left(\begin{array}{cc}
\frac{\dot{x}}{x}+\frac{1}{x}+\frac{\dot{r}}{r} & \sqrt{-2 \eta} \\
\sqrt{-2 \eta)} & -\frac{\dot{x}}{x}-\frac{1}{x}-\frac{\dot{r}}{r}
\end{array}\right), \\
M & =\left(\begin{array}{cc}
0 & -\frac{x r(t)}{2 \sqrt{-2 \eta}} \\
\frac{x r(t)}{2 \sqrt{-2 \eta}} & 0
\end{array}\right) .
\end{aligned}
$$

The quadratic first integral follows by the usual formula and is given by

$$
I_{15}=\left(\frac{\dot{x}}{x}+\frac{1}{x}+\frac{\dot{r}}{r}\right)^{2}-2 x r(t)-\int r(t) d t
$$

A more or less similar procedure also yields the Lax pair for equation No XVI, which is stated below:

\section{Equation XVI}

$$
\ddot{x}=\frac{1}{x} \dot{x}^{2}-\dot{q}(t) \frac{\dot{x}}{x}+x^{3}-q(t) x^{2}+\ddot{q}(t) .
$$

The Lax pair is

$$
\begin{aligned}
L & =\left(\begin{array}{cc}
\frac{\dot{x}}{x}-\frac{\dot{q}(t)}{x} & \sqrt{-(x-q(t))^{2}} \\
\sqrt{-(x-q(t))^{2}} & -\frac{\dot{x}}{x}+\frac{\dot{q}(t)}{x}
\end{array}\right), \\
M & =\left(\begin{array}{cc}
0 & i \frac{x}{2} \\
-i \frac{x}{2} & 0
\end{array}\right) .
\end{aligned}
$$

The quadratic first integral follows by the usual formula and is given by

$$
I_{16}=\left(\frac{\dot{x}}{x}-\frac{q(t)}{x}\right)^{2}-(x-q(t))^{2} .
$$

REMARK. As for some of the other non-autonomous equations of Gambier's list such as equations XIV, XX and XXXIV which are of the above type, the chief difficulty appears to be in expressing the explicitly time-dependent part of the equation as a function of the characteristic coordinate $\eta$.

\subsection{Autonomous Painlevé-Gambier equations}

From the dynamical point of view the existence of time-independent first integrals is much more useful as they allow for a more physical interpretation. The procedure for deriving Lax pairs for ordinary differential equations as outlined in the previous sections led to equations which were manifestly non-autonomous. However, as explained in the next section, one can also derive time-independent first integrals, using a Lax representation, for a particular class of autonomous ordinary differential equations of the form

$$
\ddot{x}+f(x) \dot{x}^{2}+g(x)=0 .
$$


Such equations may be referred to as the Liénard equations of the second kind as they involve a quadratic dependence on the velocity. An interesting example of such an equation in dynamical systems is provided by the Mathews - Lakshmanan equation

$$
\left(1+\lambda x^{2}\right) \ddot{x}-\lambda x \dot{x}^{2}+\alpha^{2} x=0, \quad \lambda>0 .
$$

Its Lax representation (the derivation of which will be clear from the next section) is given by

$$
L=\left(\begin{array}{cc}
\frac{\dot{x}}{\sqrt{1+\lambda x^{2}}} & \frac{i \alpha}{\sqrt{\lambda\left(1+\lambda x^{2}\right)}} \\
\frac{i \alpha}{\sqrt{\lambda\left(1+\lambda x^{2}\right)}} & -\frac{\dot{x}}{\sqrt{1+\lambda x^{2}}}
\end{array}\right), \quad M=\left(\begin{array}{cc}
0 & \frac{i \alpha \sqrt{\lambda} x}{2\left(1+\lambda x^{2}\right)} \\
-\frac{i \alpha \sqrt{\lambda} x}{2\left(1+\lambda x^{2}\right)} & 0
\end{array}\right)
$$

and the equation admits the first integral

$$
I(x, \dot{x})=\frac{\lambda \dot{x}^{2}-\alpha^{2}}{\lambda\left(1+\lambda x^{2}\right)} .
$$

In the following section we show that it is possible to derive in a simple manner time-independent first integrals for several equations of the Painlevé-Gambier list by employing the Lax representation for these equations.

Many equations of the Painlevé-Gambier list are characterised by the absence of the damping term, i.e., the term involving $\dot{x}$, but depend on $\dot{x}^{2}$. These equations are typically of the form stated in (3.1). For such autonomous equations we find that we can drop the function $F$ in the Lax operator $L$ and consider a generic form given by

$$
L=\left(\begin{array}{cc}
m(x) \dot{x} & U(x) \\
U(x) & -m(x) \dot{x}
\end{array}\right), \quad M=\left(\begin{array}{cc}
0 & B \\
-B & 0
\end{array}\right) .
$$

By following a procedure similar to that discussed in the earlier sections one finds from the Lax equation that

$$
m(x)=e^{\int f(x) d x}, \quad \frac{1}{2 m^{2}(x)} \frac{\partial}{\partial x}\left(U^{2}\right)=g(x), \quad B(x)=\frac{U_{x}}{2 m(x)} .
$$

We present below a list of Painleve-Gambier type equations [7] belonging to this category together with their corresponding Lax pairs and associated quadratic first integral calculated by means of the formula $I_{2}=\operatorname{tr}\left(L^{2}\right) / 2$ :

\section{Equation XII:}

$$
\begin{aligned}
& \ddot{x}=\frac{1}{x} \dot{x}^{2}+\alpha x^{3}+\beta x^{2}+\gamma+\frac{\delta}{x}, \\
& L=\left(\begin{array}{cc}
\frac{\dot{x}}{x} & i\left(\alpha x^{2}+2 \beta x-\frac{2 \gamma}{x}-\frac{\delta}{x^{2}}\right)^{1 / 2} \\
i\left(\alpha x^{2}+2 \beta x-\frac{2 \gamma}{x}-\frac{\delta}{x^{2}}\right)^{1 / 2} & -\frac{\dot{x}}{x}
\end{array}\right) \text {, } \\
& M=\left(\begin{array}{cc}
0 & \frac{i}{2} \frac{\left(\alpha x^{2}+\beta x+\frac{\gamma}{x}+\frac{\delta}{x^{2}}\right)}{\sqrt{\left(\alpha x^{2}+2 \beta x-\frac{2 \gamma}{x}-\frac{\delta}{x^{2}}\right)}} \\
-\frac{i}{2} \frac{\left(\alpha x^{2}+\beta x+\frac{\gamma}{x}+\frac{\delta}{x^{2}}\right)}{\sqrt{\left(\alpha x^{2}+2 \beta x-\frac{2 \gamma}{x}-\frac{\delta}{x^{2}}\right)}} & 0
\end{array}\right), \\
& I_{2}^{12}=\frac{\dot{x}^{2}}{x^{2}}-\left(\alpha x^{2}+2 \beta x-\frac{2 \gamma}{x}-\frac{\delta}{x^{2}}\right) .
\end{aligned}
$$


Equation XVIII:

$$
\begin{gathered}
\ddot{x}=\frac{1}{2 x} \dot{x}^{2}+4 x^{2}, \\
L=\left(\begin{array}{cc}
\frac{\dot{x}}{\sqrt{x}} & 2 i x \\
2 i x & -\frac{\dot{x}}{\sqrt{x}}
\end{array}\right), \quad M=\left(\begin{array}{cc}
0 & i \sqrt{x} \\
-i \sqrt{x} & 0
\end{array}\right), \\
I_{2}^{18}=\frac{\dot{x}^{2}}{x}-4 x^{2} .
\end{gathered}
$$

Equation XIX:

$$
\begin{gathered}
\ddot{x}=\frac{1}{2 x} \dot{x}^{2}+4 x^{2}+2 x, \\
L=\left(\begin{array}{cc}
\frac{\dot{x}}{\sqrt{x}} & 2 i \sqrt{x(x+1)} \\
2 i \sqrt{x(x+1)} & -\frac{\dot{x}}{\sqrt{x}}
\end{array}\right), \quad M=\left(\begin{array}{cc}
0 & \frac{i}{2} \frac{2 x+1}{\sqrt{x+1}} \\
-\frac{i}{2} \frac{2 x+1}{\sqrt{x+1}} & 0
\end{array}\right), \\
I_{2}^{19}=\frac{\dot{x}^{2}}{x}-4 x(x+1) .
\end{gathered}
$$

Equation XXI:

$$
\begin{gathered}
\ddot{x}=\frac{3}{4 x} \dot{x}^{2}+3 x^{2}, \\
L=\left(\begin{array}{cc}
x^{-3 / 4} \dot{x} & 2 i x^{3 / 4} \\
2 i x^{3 / 4} & -x^{-3 / 4} \dot{x}
\end{array}\right), \quad M=\left(\begin{array}{cc}
0 & \frac{3 i}{4} x^{1 / 2} \\
-\frac{3 i}{4} x^{1 / 2} & 0
\end{array}\right), \\
I_{2}^{21}=\frac{\dot{x}^{2}}{x^{-3 / 2}}-\frac{4}{x^{3 / 2}} .
\end{gathered}
$$

Equation XXII:

$$
\begin{gathered}
\ddot{x}=\frac{3}{4 x} \dot{x}^{2}-1 \\
L=\left(\begin{array}{cc}
x^{-3 / 4} \dot{x} & 2 i x^{-1 / 4} \\
2 i x^{-1 / 4} & -x^{-3 / 4} \dot{x}
\end{array}\right), \quad M=\left(\begin{array}{cc}
0 & -\frac{i}{4} x^{-1 / 2} \\
\frac{i}{4} x^{-1 / 2} & 0
\end{array}\right), \\
I_{2}^{22}=\frac{\dot{x}^{2}}{x^{3 / 2}}-\frac{4}{x^{1 / 2}} .
\end{gathered}
$$

Equation XXIII:

$$
\begin{gathered}
\ddot{x}=\frac{3}{4 x} \dot{x}^{2}+3 x^{2}+\alpha x+\beta, \\
L=\left(\begin{array}{cc}
x^{-3 / 4} \dot{x} & \frac{2 i}{x^{1 / 4}}\left(x^{2}+\alpha x-\beta\right)^{1 / 2} \\
\frac{2 i}{x^{1 / 4}}\left(x^{2}+\alpha x-\beta\right)^{1 / 2} & -x^{-3 / 4} \dot{x}
\end{array}\right), \\
M=\left(\begin{array}{cc}
0 & \frac{i}{4} \frac{3 x^{2}+\alpha x+\beta}{\left(x^{3}+\alpha x^{2}-\beta x\right)^{1 / 2}} \\
-\frac{i}{4} \frac{3 x^{2}+\alpha x+\beta}{\left(x^{3}+\alpha x^{2}-\beta x\right)^{1 / 2}} & 0
\end{array}\right), \\
I_{2}^{23}=\frac{\dot{x}^{2}}{x^{3 / 2}}-\frac{4}{x^{1 / 2}}\left(x^{2}+\alpha x-\beta\right) .
\end{gathered}
$$

H) — RUSSIAN JOURNAL OF NONLINEAR DYNAMICS, 2020, 16(4), 637-650 _ 


\section{Equation XXIX:}

Equation XXXII:

$$
\begin{gathered}
\ddot{x}=\frac{1}{2 x} \dot{x}^{2}+\frac{3}{2} x^{3}, \\
L=\left(\begin{array}{cc}
\frac{\dot{x}}{\sqrt{x}} & i x^{3 / 2} \\
i x^{3 / 2} & -\frac{\dot{x}}{\sqrt{x}}
\end{array}\right), \quad M=\left(\begin{array}{cc}
0 & \frac{3 i}{4} x \\
-\frac{3 i}{4} x & 0
\end{array}\right), \\
I_{2}^{29}=\frac{\dot{x}^{2}}{x}-x^{3} .
\end{gathered}
$$

$$
\begin{gathered}
\ddot{x}=\frac{1}{2 x} \dot{x}^{2}-\frac{1}{2 x}, \\
L=\left(\begin{array}{cc}
\frac{\dot{x}}{\sqrt{x}} & \frac{i}{\sqrt{x}} \\
\frac{i}{\sqrt{x}} & -\frac{\dot{x}}{\sqrt{x}}
\end{array}\right), \quad M=\left(\begin{array}{cc}
0 & -\frac{i}{4 x} \\
\frac{i}{4 x} & 0
\end{array}\right), \\
I_{2}^{32}=\frac{\dot{x}^{2}}{x}-\frac{1}{x} .
\end{gathered}
$$

\subsection{Autonomous equations of the form $\ddot{x}=f(x) \dot{x}^{2}$}

These include equations XVII, XXXVII, XLI, XLIII among others [7]. In these cases we find that the function $U=U(\dot{x})$, i.e, all entries of $L$ depend on $\dot{x}$, as do the entries of the matrix $M$. We list below the respective Lax pairs.

\section{Equation XVII}

$$
\begin{gathered}
\ddot{x}=\frac{m-1}{m x} \dot{x}^{2}, \quad m=\text { const, } \\
L=\left(\begin{array}{cc}
\left(\sqrt{\left.x^{-2(m-1) / m}-1\right)} \dot{x}\right. & \dot{x} \\
\dot{x} & -\left(\sqrt{x^{-2(m-1) / m}-1}\right) \dot{x}
\end{array}\right), \\
M=\left(\begin{array}{cc}
0 & \frac{m-1}{2 m x} \frac{1}{\sqrt{x^{-2(m-1) / m}-1}} \dot{x} \\
-\frac{m-1}{2 m x} \frac{1}{\sqrt{x^{-2(m-1) / m}-1}} \dot{x} & 0
\end{array}\right) .
\end{gathered}
$$

The first integral is

$$
I=\frac{\dot{x}^{2}}{x^{2(m-1) / m}}
$$

Equation XXXVII

$$
\begin{gathered}
\ddot{x}=\frac{3}{4}\left(\frac{1}{2 x}+\frac{1}{x-1}\right) \dot{x}^{2}, \\
L=\left(\begin{array}{cc}
\left(\sqrt{\frac{1}{x(x-1)^{2}}-1}\right) \dot{x} & \dot{x} \\
\dot{x} & -\left(\sqrt{\frac{1}{x(x-1)^{2}}-1}\right) \dot{x}
\end{array}\right), \\
M=\left(\begin{array}{cc}
0 & \frac{3 x-1}{4 \sqrt{x-x^{2}(x-1)^{2}}} \dot{x} \\
-\frac{3 x-1}{4 \sqrt{x-x^{2}(x-1)^{2}}} \dot{x} & 0
\end{array}\right) .
\end{gathered}
$$

The first integral is

$$
I=\frac{\dot{x}^{2}}{x(x-1)^{2}}
$$




\section{Equation XLI}

$$
\begin{gathered}
\ddot{x}=\frac{2}{3}\left(\frac{1}{x}+\frac{1}{x-1}\right) \dot{x}^{2}, \\
L=\left(\begin{array}{cc}
\left(\sqrt{\frac{1}{\left(x^{2}-x\right)^{4 / 3}}-1}\right) \dot{x} & \dot{x} \\
\dot{x} & -\left(\sqrt{\frac{1}{\left(x^{2}-x\right)^{4 / 3}}-1}\right) \dot{x}
\end{array}\right), \\
0 \\
0=\left(\begin{array}{cc}
\frac{2 x-1}{3 x^{1 / 3}(x-1)^{1 / 3} \sqrt{1-\left(x^{2}-x\right)^{4 / 3}}} \dot{x} \\
-\frac{\left.1 x^{2}-x\right)^{4 / 3}}{3 x^{1 / 3}(x-1)^{1 / 3} \sqrt{1-x^{2}}}
\end{array}\right) .
\end{gathered}
$$

The first integral is

$$
I=\frac{\dot{x}^{2}}{\left(x^{2}-x\right)^{4 / 3}} .
$$

\section{Equation XLIII}

$$
\begin{gathered}
\ddot{x}=\frac{3}{4}\left(\frac{1}{x}+\frac{1}{x-1}\right) \dot{x}^{2}, \\
L=\left(\begin{array}{cc}
\left(\sqrt{\left(x^{2}-x\right)^{-3 / 2}-1}\right) \dot{x} & \dot{x} \\
\dot{x} & -\left(\sqrt{\left(x^{2}-x\right)^{-3 / 2}-1}\right) \dot{x}
\end{array}\right), \\
M=\left(\begin{array}{cc}
0 & \frac{3}{8} \frac{2 x-1}{\left(x^{2}-x\right) \sqrt{\left(x^{2}-x\right)^{-3 / 2}-1}} \dot{x} \\
-\frac{3}{8} \frac{2 x-1}{\left(x^{2}-x\right) \sqrt{\left(x^{2}-x\right)^{-3 / 2}-1}} \dot{x} & 0
\end{array}\right) .
\end{gathered}
$$

The first integral is

$$
I=\frac{\dot{x}^{2}}{\left(x^{2}-x\right)^{3 / 2}}
$$

\section{Conclusion}

In this paper we have extended the previous studies of the Lax formulation of non-autonomous equations of Liénard type by Sinelshchikov et al. [1] to non-autonomous equations including an additional term depending quadratically on the velocity, namely, the Levinson-Smith equation. The first integrals resulting from the trace formula of the Lax pair for such equations are invariably time-dependent. In particular, we have obtained the Lax pair and first integrals for the non-autonomous equations numbered XV and XVI as given in [7]. However, it is found that for the non-autonomous equations XIV, XX and XXXIV we are unable to deduce the Lax pair chiefly because of our apparent inability to write the time-dependent part of the equation in terms of an appropriate function of the characteristic coordinate $\eta$. Nevertheless, we have shown that no such difficulty arises for the autonomous ODEs of the Painlevé-Gambier list and have explicitly derived the Lax representations for equations Nos. XII, XVII, XVIII, XIX, XXI, XXII, XXIII, XXIX, XXXII, XXXVII, XLI and XLIII of this list. In these cases the existence of a Lax representation leads to quadratic first integrals which are inevitably time-independent. Note that such first integrals may be considered as an analog of the energy conservation law for the dissipative systems and the equations themselves may be physically interpreted as representing position-dependent mass systems.

An interesting issue related to the results of this article concerns their generalisation to high-order equations. Rational solutions of the Painlevé equations (also hierarchy) are important 
features in integrable systems. Kudryashov and his coworkers [9-12] have in fact focussed on this issue and by extending the method of Weiss [13] derived rational solutions of the members of the Painlevé II, in particular, the second member of the hierarchy, which leads to a fourth-order ODE.

Finally, it may be remarked that in one of our earlier works we had obtained non-quadratic first integrals for a subset of equations belonging to the Painlevé-Gambier classification using the method of (generalised) Sundman transformations (GST)[8], where we considered the condition for linearisability of the non-linear equations via a GST. However, the procedure described in this paper allows us to obtain quadratic first integrals of the Painlevé-Gambier equations in a more elegant manner using their Lax representations.

\section{Acknowledgments}

We are immensely grateful to Dimitry Sinelshchikov for various discussions and correspondences. We wish to thank Professor Basil Grammaticos for enlightening discussions and constant encouragements to pursue research on the Painlevé-Gambier equations even a century after their appearance. Finally, we thank the anonymous referee for drawing our attention to references [9-12].

\section{Conflict of Interest}

The authors declare that they have no conflict of interest.

\section{References}

[1] Sinelshchikov, D. I., Gaiur, I. Yu., and Kudryashov, N. A., Lax Representation and Quadratic First Integrals for a Family of Non-Autonomous Second-Order Differential Equations, J. Math. Anal. Appl., 2019, vol. 480, no. 1, 123375, 14 pp.

[2] Adler, M. and van Moerbeke, P., Completely Integrable Systems, Euclidean Lie Algebras, and Curves, Adv. in Math., 1980, vol.38, no. 3, pp. 267-317.

[3] Griffiths, Ph. A., Linearizing Flows and a Cohomological Interpretation of Lax Equations, Amer. J. Math., 1985, vol. 107, no. 6, pp. 1445-1484.

[4] Ravoson, V., Gavrilov, L., and Caboz, R., Separability and Lax Pairs for Hénon-Heiles System, J. Math. Phys., 1993, vol. 34, no. 6, pp. 2385-2393.

[5] Fairbanks, L. D., Lax Equation Representation of Certain Completely Integrable Systems, Compositio Math., 1988, vol. 68, no. 1, pp. 31-40.

[6] Kudryashov, N.A. and Sinelshchikov, D. I., On Connections of the Liénard Equation with Some Equations of Painlevé-Gambier Type, J. Math. Anal. Appl., 2017, vol. 449, no. 2, pp. 1570-1580.

[7] Ince, E. L., Ordinary Differential Equations, New York: Dover, 1956.

[8] Guha, P., Khanra, B., and Choudhury, A. Gh., On Generalized Sundman Transformation Method, First Integrals, Symmetries and Solutions of Equations of Painlevé-Gambier Type, Nonlinear Anal., 2010, vol. 72, nos. 7-8, pp. 3247-3257.

[9] Kudryashov, N.A. and Pickering, A., Rational Solutions for Schwarzian Integrable Hierarchies, J. Phys. A, 1998, vol. 31, no. 47, pp. 9505-9518.

[10] Kudryashov, N. and Soukharev, M., Uniformization and Transcendence of Solutions for the First and Second Painlevé Hierarchies, Phys. Lett. A., 1998, vol. 237, nos. 4-5, pp. 206-216.

RUSSIAN JOURNAL OF NONLINEAR DYNAMICS, 2020, 16(4), 637-650 
[11] Kudryashov, N. A., Two Hierarchies of Ordinary Differential Equations and Their Properties, Phys. Lett. A, 1999, vol. 252, pp. 173-179.

[12] Kudryashov, N. A., Transcendents Defined by Nonlinear Fourth-Order Ordinary Differential Equations, J. Phys. A., 1999, vol. 32, no. 6, pp. 999-1013.

[13] Weiss, J., The Painlevé Property for Partial Differential Equations: 2. Bäcklund Transformation, Lax Pairs, and the Schwarzian Derivative, J. Math. Phys., 1983, vol. 24, no. 6, pp. 1405-1413. 